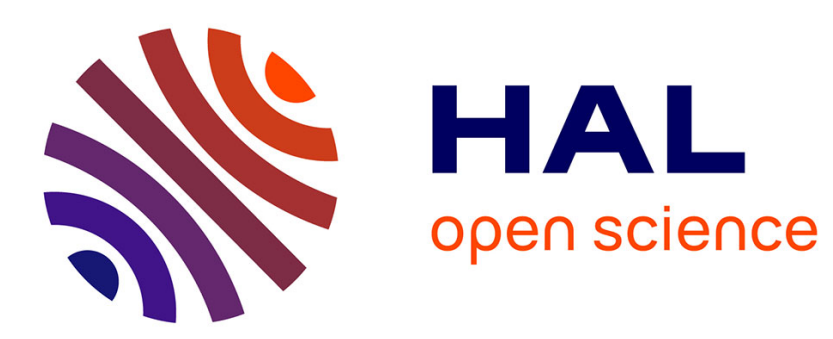

\title{
Influence of the Silica Support on the Structure and the Morphology of Silver Nanoparticles: A Molecular Simulation Study
}

Alain Cabrel Ngandjong, C. Mottet, Joël Puibasset

\section{- To cite this version:}

Alain Cabrel Ngandjong, C. Mottet, Joël Puibasset. Influence of the Silica Support on the Structure and the Morphology of Silver Nanoparticles: A Molecular Simulation Study. Journal of Physical Chemistry C, 2016, 120 (15), pp.8323-8332. 10.1021/acs.jpcc.6b00290 . hal-01459056

\author{
HAL Id: hal-01459056 \\ https://hal.science/hal-01459056
}

Submitted on 11 Dec 2020

HAL is a multi-disciplinary open access archive for the deposit and dissemination of scientific research documents, whether they are published or not. The documents may come from teaching and research institutions in France or abroad, or from public or private research centers.
L'archive ouverte pluridisciplinaire HAL, est destinée au dépôt et à la diffusion de documents scientifiques de niveau recherche, publiés ou non, émanant des établissements d'enseignement et de recherche français ou étrangers, des laboratoires publics ou privés. 


\section{Influence of the Silica Support on the Structure and the Morphology of}

\section{Silver Nanoparticles: a Molecular Simulation Study}

A. C. Ngandjong ${ }^{1}$, C. Mottet ${ }^{2}$, J. Puibasset ${ }^{1 *}$

${ }^{1}$ Interfaces, Confinement, Matériaux et Nanostructures (ICMN), UMR 7374, CNRS et Université d'Orléans, $1 \mathrm{~b}$ rue de la Férollerie, 45071 Orléans

${ }^{2}$ Aix-Marseille Université, CNRS, CINaM UMR7325, Campus de Luminy, case 913, 13288 Marseille, Cedex 9, France

Electronic-mails: angandjong@cnrs-orleans.fr, mottet@cinam.univ-mrs.fr, puibasset@cnrsorleans.fr

Corresponding author: E-mail: puibasset@cnrs-orleans.fr; Tel: 33238255803 


\section{Abstract:}

We investigate the effect of the support on the structure and morphology of deposited metallic nanoparticles via molecular simulations at zero temperature. We focus on silver clusters in contact with a substrate which exhibits weak interactions, as amorphous silica, frequently used in atomic deposition experiments. Three levels of approximation are used to describe the substrate: (i) the smooth wall approximation where the support is modeled by a square-well whose depth is related to the adhesion energy of the nanoparticle, (ii) an atomistic model of flat amorphous silica obtained from Monte Carlo simulations with effective pair potentials, and (iii) the same silica substrate partially melted in order to roughen the surface at the nanometric scale. The metal-metal interactions are modeled using tight-binding semi-empirical potentials, and the metal-silica interactions are modeled with Lennard-Jones potentials fitted to $a b$-initio or experimental data. The icosahedron/decahedron/truncated octahedron sequence found for free $\mathrm{Ag}$ nanoparticles is shown to be affected by the substrate (square-well), which favors truncated octahedrons deposited on their (111) facets. The aspect ratio is shown to follow the Wulff-Kaishew theorem for large clusters. The introduction of an atomistic description of the flat $\mathrm{SiO}_{2}$ surface does not change the results significantly, while the presence of a nanometric roughness induces drastic changes. Vertex orientations of nanoparticles are possible in the surface hollows, and decahedron structures are slightly favored for sizes smaller than 1200 atoms. 


\section{INTRODUCTION}

Supported metallic nanoparticles on oxide substrates are the systems of choice to study model catalysts $^{1-7}$ using surface science experimental setups like grazing incidence $\mathrm{X}$-ray scattering ${ }^{8}$ or (high resolution) transmission electron microscopy ${ }^{9}$ to characterize the structure and morphology of the nanoparticles. The nanoparticles are either grown directly on a surface in ultrahigh vacuum conditions ${ }^{10}$ or produced in a molecular beam and then deposited on a substrate $^{11}$. In both cases, an oxide support is chosen in order to minimize the interaction with the metallic clusters in order to keep a three-dimensional morphology as much as comparable to the one of the cluster free of interaction. Depending on the nature of the substrate, its effect on the structure and morphology of the nanoparticles will be different. In the case of crystalline substrates like $\mathrm{MgO}(100)$ surface, it has been shown that the substrate can modify the atomic structure and morphology of the supported metal nanoparticles. ${ }^{9}$ Whereas unsupported nanoparticles can adopt non-periodic structures (icosahedral or decahedral symmetries) ${ }^{11}$, they change their structure to the face centered cubic structure when grown on $\mathrm{MgO}(100)$ surface as observed by $x$-ray scattering ${ }^{12}$ or by electron microscopy ${ }^{13}$, and confirmed by numerical simulations. ${ }^{14-19}$ In the case of amorphous substrates like silica or carbon, the interactions with the deposited nanoparticles are much weaker and these supports are often used in experiments to minimize surface effects in order to preserve the properties of the free nanoparticles. Even if the interactions are weak, experiments suggest however some effects which have to be considered to get a precise characterization of both the morphology and the structure of nanoparticles. ${ }^{20,21}$ This has been the motivation of this study, also because from the theoretical 
point of view, there is much less study concerning the simulation of nanoparticle in interaction with amorphous support.

This weak interaction type is essentially characterized by van der Waals interactions, even though some unsaturated surface atoms can induce strong interactions (specific sites). Indeed, amorphous substrates, and especially hydroxylated silica surface, are complex systems where both the chemistry and the disordered structure make it difficult to model. ${ }^{22,23}$ There are either empirical potentials to describe crystalline or amorphous silica ${ }^{24-27}$ or $a b$ initio density functional theory (DFT) calculations on various silica crystals and surfaces, ${ }^{28-31}$ but much less DFT studies of the amorphous silica. ${ }^{32-34}$

A quantitative analysis of substrate effect cannot be too general. We first focus on the structural stability of the nanoparticle, as an important example of physical property. We also have to choose a specific system, since the structural stability of free nanoparticles is highly dependent on the metal, and possibly, for a given metal, on the choice of the potential. We focus on silver deposited on amorphous silica because this system has already attracted interest in the past and has been experimentally studied in our team. Silver nanoparticles present interesting properties in optics thanks to a strong localized surface-plasmon resonance in the absorption spectra leading to many applications in plasmonics, ${ }^{35,36}$ surface-enhanced spectroscopies ${ }^{37}$ or bio-sensors ${ }^{38}$. These properties highly depend on the shape and size of the nanoparticles which are influenced themselves by their support. In this work, we use well-known semi-empirical potentials for the metal cluster and the amorphous silica, and a van der Waals like (Lennard-Jones) potential fitted to DFT and experimental results for the metal-silica interaction. 
Since we focus on the influence of the support, we first discard the temperature effects. We thus compare only energies, and the obtained results are actually valid only for zero temperature. As usual when comparing the tiny energy differences between nanoparticles of different structure and similar sizes, we introduce the so-called excess energy $\Delta$ :

$$
\Delta=\frac{E-N e}{N^{2 / 3}}
$$

where $E$ is the nanoparticle energy, $N$ its number of atoms, and $e$ the bulk cohesion energy of the metal at the same temperature (zero in our case). The determination of the nanoparticle's structure at equilibrium and at low temperature remains a challenging task, because the number of energy minima increases exponentially with size. ${ }^{39}$ This is even more difficult in presence of a substrate, even rigid, in particular if different adsorption sites have to be considered. Our approach consists in considering that the equilibrium configurations of supported nanoparticles on a weak substrate are most probably not so different from the wellknown equilibrium structures for free nanoparticles. It is then possible to guess reasonable structures for supported nanoparticles which would derive from the free equilibrium configurations (by removing atomic layers at the metal-substrate interface to change the aspect ratio for instance). This avoids a time consuming systematic exploration. We may however miss some exotic configurations.

This paper is structured as follows: we first present the numerical details associated with the interatomic potentials used. Then we present the three support models used in this study: the smooth wall approximation with a square-well potential, and two fully atomistic silica substrates, flat and rough. They are characterized in terms of energetic profile. The third section 
presents the energy minimizations for various deposited nanoparticles. The effect of interaction intensity and surface roughness are considered and discussed.

\section{METHODS}

This work focuses on the determination of equilibrium configurations at zero temperature for metallic nanoparticles comprising several thousands of atoms. Our molecular approach will thus be limited to energy minimizations using semiempirical interatomic potentials. The most simple and efficient enough algorithm in this case is the microcanonical quenched molecular dynamics which consists in setting to zero any atomic velocity which verifies $\boldsymbol{v}_{i} . \boldsymbol{f}_{i}<0$ where $\boldsymbol{v}_{i}$ and $\boldsymbol{f}_{i}$ are the velocity and the force acting on atom $i$. We have also sometimes performed Monte Carlo runs where the temperature was progressively reduced to zero. We used the Metropolis algorithm ${ }^{40}$ where the atomic displacements are accepted if the total energy of the system is lowered; conversely, when the total energy increases, the displacement is accepted according to the Boltzmann weight. This algorithm ensures to get a Boltzmann distribution of the equilibrium configuration. This method is expected to be more efficient to explore all the metastable configurations in the neighborhood of the initial one, i.e. accessible through simple atomic displacements. In practice,

no improvement was obtained for the structure of the nanoparticle itself, but some improvement was sometimes possible in terms of nanoparticle position on the atomic silica surface which presents inhomogeneity. 


\subsection{Metal-metal interaction}

The metal-metal interactions are modeled by the semi-empirical many-body potential derived from the tight binding scheme in the second moment approximation (TBSMA). ${ }^{41}$ The energy at site " $n$ " writes in two terms. An attractive many-body term coming from the band energy in the form:

$$
\mathrm{E}_{\mathrm{n}}^{\mathrm{b}}=-\sqrt{\sum_{r_{n m}<r_{c}}^{m \neq n} \xi^{2} \exp \left[-2 q\left(\frac{r_{n m}}{r_{0}}-1\right)\right]}
$$

where " $r \mathrm{~nm}$ " is the interatomic distance between atom at site " $n$ " and its neighbor at site " $\mathrm{m}$ ", with $r_{n m}<r_{c}$, the cutoff distance equivalent to the distance of the second neighbors in bulk Ag, $r_{0}$ is the first-neighbor distance. The second term is a core-repulsion term of the Born-Mayer type:

$$
\mathrm{E}_{\mathrm{n}}^{\mathrm{r}}=\sum_{r_{n m}<r_{c}} A \exp \left[-p\left(\frac{r_{n m}}{r_{0}}-1\right)\right]
$$

where $\mathrm{p}=10.790, \mathrm{q}=3.190, \mathrm{~A}=0.10433 \mathrm{eV}$ and $\xi=1.19402 \mathrm{eV}$ are four parameters fitted to the bulk cohesive energy, lattice parameter and elastic constants. ${ }^{17,42}$ After the second neighbors, the potential is continuously prolonged up to zero by a fifth order polynomial form to cancel the interaction after the third neighbor distance.

\subsection{Silica species interaction}

The silica substrate is modeled with the m-TTAM potential. ${ }^{24,26}$ The TTAM ${ }^{24}$ potential developed by Tsuneyuki, Tsukada, Aoki and Matsui is based on the idea that the local tetrahedral 
structures present in silica can be reproduced with two-body spherically symmetric potentials acting on $\mathrm{Si}^{4+}$ and $\mathrm{O}^{2-}$ ions. The analytical form of the potential if of Born-Huggins-Mayer type, including electrostatics, soft core and dispersion forces. This potential is very simple to implement in a molecular dynamics simulation, and has proven to be accurate enough to describe the various crystalline polymorphs of silica. At high temperature, the high kinetic energy of the atoms allows them to occasionally override the atom-atom repulsion barrier at very short range where the dispersive term spuriously dominates. Guissani and Guillot ${ }^{26}$ cured this problem by adding a $r^{-18}$ repulsive term, and thus improved the potential at high temperature (up to $5000 \mathrm{~K}$ ). This potential is used to elaborate our amorphous silica support, which is then kept rigid when the metallic nanoparticles are introduced. In principle, the creation of a free surface creates highly reactive dangling bonds like $\mathrm{Si}-\mathrm{O}^{\circ}$, which have to be saturated with hydrogen to form hydroxyl groups Si-O-H. This procedure mimics a realistic silica surface which has been put in contact with air, as is the case for metal deposition experiments. This is also a way to compensate the polarity of the oxide surface ${ }^{43}$ induced by the charged ionic species at the surface. The metal-silica interaction, to be described now, takes this point into account in an effective approach, i.e. the hydrogen atom is not described explicitly: the hydroxyl group is modeled as a point-like interaction site.

\subsection{Metal-silica interaction}

The silver-silica interaction is not so well documented. Experiments show that metallic nanoparticles interact weakly with amorphous silica substrate. For instance, liquid drop 
measurements performed by Eusthatopoulos and coworkers $s^{44,45}$ give a wetting angle of $142^{\circ}$. $A b$ initio calculations performed on a similar system, silver on a silica crystal ( $\alpha$-quartz) also show that interactions are weak. ${ }^{46}$ In order to complete these results we have done ab initio calculations for silver deposited on another silica crystal, the $\beta$-cristobalite, whose density is close to that of the amorphous silica. Such calculations were successfully used by Nagao et al. for the $\mathrm{Cu} / \mathrm{SiO}_{2}$ system. ${ }^{47}$ The choice of a crystal is imposed by the difficulty to model amorphous silica with ab initio methods.

The DFT calculations are performed using the Vienna Ab initio Simulation Package (VASP) code and the projector augmented wave (PAW) $)^{48,49}$ interaction potential between the electrons and the ions. The exchange and correlation functional is the generalized gradient approximation (GGA-PBE). ${ }^{50}$ The Brillouin zone integration is performed with the Monkhorst-Pack scheme with $k$-point mesh comprising 10 subdivisions along each reciprocal lattice vector and only one subdivision in the direction perpendicular to the surface. The energy cutoff is taken equal to 450 $\mathrm{eV}$ for each type of atom. The lattice parameter of the $\beta$-cristobalite is found to be equal to 7.46 Angströms (to be compared with the experimental value of 7.16 Angströms). For the (001) surface of $\beta$-cristobalite, we considered one initial slab of $8 \mathrm{Si}$ atoms and $16 \mathrm{O}$ atoms. The surface is then fully hydroxylated by saturating $\mathrm{Si}$ with $\mathrm{OH}$ groups and $\mathrm{O}$ with $\mathrm{H}$ atoms. This leads to add $4 \mathrm{H}_{2} \mathrm{O}$ molecules to our slab. The corresponding surface density of hydroxyl groups is $7.2 \mathrm{OH} / \mathrm{nm}^{2}$. We also considered a partially hydroxylated surface corresponding to a coverage of $3.6 \mathrm{OH} / \mathrm{nm}^{2}$, closer to experimental measurements, ${ }^{23}$ by removing $2 \mathrm{H}$ and $2 \mathrm{OH}$ (silanisation). Then we considered three different metal deposits: one single Ag adatom (per $\mathrm{SiO}_{2}$ mesh which means with a distance of $0.746 \mathrm{~nm}$ between the $\mathrm{Ag}$ atoms), one monolayer 
(i.e. $8 \mathrm{Ag}$ atoms per $\mathrm{SiO}_{2}$ mesh) and four monolayers (i.e. $32 \mathrm{Ag}$ atoms per $\mathrm{SiO}_{2}$ mesh). The results are given in the Table 1.

The calculations show that the energies involved are one order of magnitude less than covalent interactions, as long as all surface oxygen are saturated. We also notice that the intensity of the interaction varies with the degree of hydroxylation of the surface (i.e. the surface density of hydroxyl groups), a result comparable to that obtained for the $\mathrm{Cu} / \mathrm{SiO}_{2}$ system. ${ }^{47}$ For a surface hydroxyl density of $3.6 \mathrm{OH} / \mathrm{nm}^{2}$, corresponding to the experimental situation, we have obtained an adhesion energy of $-0.04 \mathrm{eV} /$ atom. This is a weak adhesion without charge transfer. To take into account the van der Waals character of the interaction, we have used a simple LennardJones interatomic potential to reproduce this adhesion energy. This potential is parameterized by a distance $(\sigma)$ and an energy $(\varepsilon)$ as follows:

$$
\mathrm{V}(r)=4 \epsilon\left[\left(\frac{\sigma}{r}\right)^{12}-\left(\frac{\sigma}{r}\right)^{6}\right]
$$

For silver-silicon and silver-oxygen we have found $\sigma_{\mathrm{Ag}-\mathrm{Si}}=0.329 \mathrm{~nm}, \varepsilon_{\mathrm{Ag}-\mathrm{Si}}=0.003 \mathrm{eV}, \sigma_{\mathrm{Ag}-\mathrm{O}}=$ $0.278 \mathrm{~nm}$, and $\varepsilon_{\mathrm{Ag}-\mathrm{O}}=0.015 \mathrm{eV}$. Note that the silver-hydrogen interaction is not described explicitly, but taken into account as an effective interaction with the $\mathrm{OH}$ groups seen as points. By definition, using these values for a silver slab deposited on cristobalite gives back the adhesion energy of $-0.04 \mathrm{eV} /$ atom found in DFT calculations.

How do these data compare with experiments? The wetting angle measurements ${ }^{44,45}$ show that the adhesion energy is closer to $-0.08 \mathrm{eV} /$ atom, a value twice larger than that found with DFT calculations. The discrepancy may originate in the fact that the dispersion contributions to the 
interaction are not taken into account in the DFT calculations we have performed. Furthermore, the systems are slightly different (crystal versus amorphous silica), and the silica surfaces used in experiments most probably exhibit reactive defects not considered in our idealized system. We thus propose to take into account these effects at a phenomenological level by considering another set of Lennard-Jones parameters where $\varepsilon_{\mathrm{Ag}-\mathrm{Si}}$ and $\varepsilon_{\mathrm{Ag}-\mathrm{o}}$ are simply multiplied by two. With this set of parameters, the adhesion energy for a silver slab deposited on cristobalite reproduces the wetting angle experiments value of $-0.08 \mathrm{eV} /$ atom. These two sets of parameters will be used to evaluate the effect of the intensity of the $\mathrm{Ag} / \mathrm{SiO}_{2}$ interaction.

\section{RESULTS AND DISCUSSION}

\subsection{Preparation and characterization of the substrates}

Metal deposition experiments are generally performed on well characterized supports like silicon wafers. These are known to exhibit a thin native oxide layer depending on its history. ${ }^{51}$ The surface is also sensitive to the presence of water when it is exposed to the air: chemical bonding of water molecules induces the formation of surface hydroxyl groups $-\mathrm{OH}$. The process is (partially) reversible, in particular in vacuum and at high temperature. ${ }^{52}$ The exact surface state thus depends on the experimental conditions, and in the conditions used for metal deposition the surface seen by the metal atoms is essentially made of hydroxylated silica. We consider three levels of approximation to model this surface. 
The simplest consists in a simple square well with short range interactions. The nanoparticle interacts only via its first layer in contact with the surface, through a uniform potential corresponding to the average adhesion energy per atom at the interface (see Fig. 1). This parameter will be varied between zero and $0.2 \mathrm{eV} /$ atom.

We then consider an atomistic model of amorphous silica. It is obtained by Monte Carlo simulation using the m-TTAM potential ${ }^{24,26}$ as follows: a silica melt initially at $2000 \mathrm{~K}$ is cooled down to $300 \mathrm{~K}$ in few steps $(1500 \mathrm{~K}, 1000 \mathrm{~K}$ and finally $300 \mathrm{~K})$. This produces a solid which is cut to form a lamella. It is then either relaxed at $300 \mathrm{~K}$, or relaxed at $2000 \mathrm{~K}$ and cooled down to 300K. In the first case, the thermal agitation does not allow large amplitude relaxations. They remain localized, even at the silica-vacuum interface. As a consequence, the lamella keeps its initial shape, with flat surfaces (see Fig 2, upper panel). In the second case, the silica lamella is allowed to melt, and the surface develops a nanometric roughness (see Fig 2 upper panel). At this level of approximation, the $\mathrm{Ag} / \mathrm{SiO}_{2}$ interactions are described by the Lennard-Jones interatomic potential given in the previous section (equation 4).

The two silica surfaces are now characterized by analyzing their interaction with an isolated silver atom which is used as a test particle above the surfaces. This characterization has been made with the potential determined from the wetting angle measurements (adhesion energy of $-0.08 \mathrm{eV} /$ atom). The potential determined from DFT calculations give identical results where all energies are divided by two. The potential felt by a silver atom is denoted $V(x, y, z)$ where $x, y$ is its position parallel to the surface and $z$ measures its height above a virtual plane passing through the surface atoms of silica and corresponding to the line of cut of the initial piece of bulk silica. Potential versus height for various imposed positions $\left(\mathrm{x}_{0}, \mathrm{y}_{0}\right)$, i.e. $\mathrm{V}\left(\mathrm{x}_{0}, \mathrm{y}_{0}, \mathrm{z}\right)$, are given 
in Fig. 2. As can be seen, the flat surface exhibits similar curves, meaning that the surface is quite homogeneous. The potential essentially presents a minimum in the vicinity of the surface, with variations in its depth. On the other hand, for the rough surface, $V\left(x_{0}, y_{0}, z\right)$ qualitatively depends on $\left(\mathrm{x}_{0}, \mathrm{y}_{0}\right)$, with eventually two minima. This behavior reveals the presence of cavities or hollows close to the surface where silver atoms may (locally) be stable.

The curves $\mathrm{V}\left(\mathrm{x}_{0}, \mathrm{y}_{0}, \mathrm{z}\right)$ present an absolute minimum, denoted $\mathrm{V}_{\min }$, for a particular height denoted $z^{*}$. Both $V_{\min }$ and $z^{*}$ depend on $x_{0}$ and $y_{0} . V_{\min }\left(x_{0}, y_{0}\right)$ actually corresponds to an adsorption energy for an imposed position $\left(\mathrm{x}_{0}, \mathrm{y}_{0}\right)$ on the surface. Its distribution is given in Fig. 2, lower panel, for both surfaces. The distribution for the rough surface is broader and shifted to lower values: more attractive sites can be found on the rough surface. In order to localize these sites, $\mathrm{V}_{\min }\left(\mathrm{x}_{0}, \mathrm{y}_{0}\right)$ is given in Fig. 3 as a color map. As can be seen, the surfaces exhibit strongly attractive valleys separated by much less attractive regions. The size of these regions corresponds to the atomic roughness for the flat surface, while they are associated with larger structures for the rough surface (nanometric surface undulations). The correlation between $V_{\min }$ and $z^{*}$ is given in Fig. 4. For the flat surface, the adsorption energy is strongly correlated with the depth $z^{*}$. This correlation is reminiscent of the weak dependence of $\mathrm{V}\left(\mathrm{x}_{0}, \mathrm{y}_{0}, \mathrm{z}\right)$ on $\mathrm{x}_{0}$ and $\mathrm{y}_{0}$. On the other hand, for the rough surface, $\mathrm{V}_{\min }$ and $\mathrm{z}^{*}$ are much less correlated, and the range of $z^{*}$ values is much larger due to the roughness.

This characterization is for an isolated silver atom adsorbed on the surface. In the case of a deposited nanoparticle, the metallic atoms in contact with the substrate belong to the same facet. The effective adhesion energy per atom is thus an average value of $V(x, y, z)$ in a plane roughly parallel to the surface. The minimum corresponds to the adhesion energy for an infinite 
silver plane. The calculations have been done for an atomistic slab of metal covering the whole surface. The results are: $-0.08 \mathrm{eV} /$ atom for the flat surface, and $-0.05 \mathrm{eV} /$ atom for the rough surface. As expected, on the flat, homogeneous surface, one recovers the adhesion energy found on cristobalite. The reason is that the density of amorphous silica is close to that of cristobalite, and the disorder associated with amorphous silica has no impact on the $\mathrm{Ag} / \mathrm{SiO}_{2}$ interaction. Surprisingly, despite the fact that the rough surface exhibits highly attractive sites, the adhesion energy is lower in intensity. These attractive sites are not necessarily accessible to a silver slab due to the geometric constrains. Note however that the highly attractive sites of the rough surface may be accessible to the smallest nanoparticles (to be discussed below).

\subsection{Structural stability of supported nanoparticles}

\subsubsection{Nanoparticles deposited on a smooth surface with the square-well potential:}

We first consider the case of a nanoparticle deposited on a smooth substrate described by the square well (see Fig. 1). If we suppose that the nanoparticle is rigid, the most favorable configuration corresponds to its largest facet in close contact with the substrate: the gained energy equals to the number of atoms of the facet times the square well depth.

Due to the surface contribution, one expects an aspect ratio of the nanoparticle different from 1. This can be seen from the Wulff-Kaishew theorem for the large nanoparticles. As a consequence, besides the most favorable free nanoparticles with sizes corresponding to magic numbers, it is important to consider also their various truncations, i.e. the removal of 1,2 or 
more atomic layers localized at the nanoparticle-substrate interface (see snapshots in Fig. 5). Two competing effects are at work: (i) removing layers induces an increase of the nanoparticle excess energy $\Delta$ defined in the introduction, as illustrated in Fig. 5, lower panel, for $e_{a d}=0$; (ii) removing layers also increases the size of the facet in contact with the substrate and thus increases the (negative) adhesion contribution to the total energy, as shown on Fig. 5, lower panel: the larger the metal-silica interface the lower the (negative) slope of the excess energy $\Delta$. Note that configurations on vertex may also become favorable after truncations. They were thus also taken into account in this study.

The calculations have thus been done for the three most stable structures in vacuum (icosahedron, Ih, Mark's decahedron, Dh, and Wulff fcc truncated octahedron, TOh), ${ }^{53-55}$ their various orientations relative to the substrate, and their successive truncations. The intensity of the metal-substrate adhesion energy per atom $e_{a d}$ has been varied between zero and 6 percent of the cohesive energy of bulk silver, $-2.95 \mathrm{eV} /$ atom. The results are summarized in Fig. 6 in a diagram where is reported the morphology of the stable nanoparticle as a function of its size and the intensity of the metal-substrate adhesion energy per atom.

The case where $e_{a d}$ is small corresponds to free nanoparticles. The icosahedron structure is shown to be the most stable for the smallest nanoparticles, followed by Mark's decahedron structure between $N=200(\sim 2 \mathrm{~nm})$ and $N=20000(\sim 8 \mathrm{~nm})$; above this size, the Wulff fcc truncated octahedron structure is the most stable. ${ }^{42,56}$ Increasing the metal-substrate interaction destabilizes the decahedron structure and favors the truncated octahedron deposited on the (111) facet (largest facet). When the interaction intensity reaches the value 
obtained by DFT calculations on the hydroxylated surface $(-0.04 \mathrm{eV} /$ atom), the Dh/TOh transition has shifted to $\mathrm{N}=1000$ atoms $(\sim 3 \mathrm{~nm})$, significantly lower than for free nanoparticles. Furthermore, favorable truncations (atomic layer removal) have appeared. On the other hand, the Ih/Dh transition has not moved whereas favorable truncations for Dh have appeared. For a larger interaction, more effects are visible: more truncations, and disappearance of Dh structures. For large nanoparticles, one recovers the Wulff-Kaishew theorem (see Fig. 6). ${ }^{57}$

\subsubsection{Nanoparticles deposited on a flat atomistic silica surface:}

The atomic silica surface model introduces a collection of interacting centers. With this model we now have lateral and vertical forces: the deposited nanoparticle is now subjected to internal constraints due to inhomogeneous forces at the nanoparticle-silica interface. The internal energy of the relaxed nanoparticle differs from its free value. Furthermore, it also depends on the exact position of the nanoparticle on the surface. We thus have to relax the nanoparticles directly on the substrate, and at the same position for all nanoparticles. This position has to be stable, i.e. corresponding to an attractive site or basin. All nanoparticles are positioned above the attractive basin in the center of the surfaces (see Fig 3). In order to optimize their final position, we first perform a Monte Carlo run at $100 \mathrm{~K}$. This temperature allows nanoparticle mobility above the surface without affecting its morphology. The temperature is then gradually reduced to zero, and the energy minimized. We have made the approximation that the substrate is not deformable, which allows to save computing time since the silica substrate is quite large compared to the nanoparticle. This is justified by the fact that the metal-silica 
interaction is quite weak compared to silica-silica interactions: the deformations of the support would thus be very small.

The minimizations have first been done for the weakest interaction intensity $(-0.04 \mathrm{eV} /$ atom), the three nanoparticle structures (Ih, Dh, TOh), their successive truncations, and various orientations (face, edge, vertex). The excess surface energy $\Delta$ is given in Fig. 7 for the TOh nanoparticle deposited on a (100) or (111) facet, and its successive truncations. The (111) orientation is more favorable (largest number of atoms), and the removal of the first layer allows to reduce the excess energy: the increase in energy of the nanoparticle is compensated by the increase in metal-silica interaction associated with the larger interface.

Figure 8 gathers the most stable configurations, in terms of orientations and truncations, for the three structures Ih, Dh and TOh. The icosahedron structure seriously disfavors truncations, and is the most favorable only for the smallest clusters. Above 150 atoms, the decahedron (which exhibits truncations) is more stable. The Ih/Dh transition is barely affected by the presence of the silica support. On the other hand, the TOh structure becomes favorable above 1000 atoms, for particles deposited on the (111) facet. The Dh/TOh transition is significantly affected by the metal-silica interaction. Inspection of the structural stability diagram (Fig. 6) shows a good agreement between the results obtained on the flat atomistic silica surface and the idealized square-well potential: the smooth wall approximation is meaningful and able to catch the main physics of nanoparticle-support interaction. This approximation is expected to be even more accurate for larger systems, and shows that even a weakly interacting atomistic support affects the morphology of a deposited nanoparticle. 


\subsubsection{Effect of the intensity of the nanoparticle-support interaction:}

The results in previous sections were obtained with the potential fitted to the DFT calculations. In order to study the effect of the interaction intensity, we now switch to the potential obtained from the wetting angle experiments, which is twice larger. For the small icosahedral structures $(\mathrm{N}<600)$, we observe that the first truncation is now more stable, for both vertex and facet orientations: in each case the gain in energy due to a larger number of atoms in contact with the substrate compensates the loss in cohesive energy due to the truncation. Calculations show that the facet orientation is the most stable between both. For the decahedral nanoparticles of size smaller than 1200 atoms, calculations show that the most favorable configurations correspond to a (100) orientation, with the removal of two metal layers in contact with the substrate. For the TOh structures of size less than 1500 atoms, the (111) orientation is favored, with removal of one or more metal layers depending on the nanoparticle size (see Fig. 9). We have evaluated the corresponding aspect ratio: $H / D=0.83$, with $15 \%$ uncertainty for the smallest sizes, down to 7\% for the largest. This value equals the expected one obtained from the Wulff-Kaishew theorem and the adhesion energy of $-0.08 \mathrm{eV} / \mathrm{at}$.

The excess energies obtained for the most favorable configurations in terms of orientation and truncation are given in Fig. 10 for the three structures Ih, Dh and TOh. The remarkable result is that TOh configurations are dominantly stable, even for nanoparticles as small as 200 atoms. Note however that the difference in excess energy with the Dh structures is tiny. Only the smallest TOh structure $(\mathrm{N}=31$, one truncation) is disfavored. In this case, the truncated $\mathrm{Dh}(\mathrm{N}=$ 
58) and $\mathrm{lh}(\mathrm{N}=49)$ structures are the most stable. Inspection of the structural stability diagram (Fig. 6) shows the same behavior for an adhesion energy of $-0.08 \mathrm{eV} /$ at: except for the smallest sizes, the stable nanoparticles are TOh structures with aspect ratio around 0.83 . Note that for larger nanoparticles, the smooth wall approximation is expected to be even more accurate, and confirms this conclusion.

\subsubsection{Nanoparticles deposited on a rough atomistic silica surface:}

The effect of the surface roughness is now studied by considering the same metal-silica interaction as before (based on wetting angle measurements) and replacing the flat silica substrate by the rough one (see Fig. 2). As previously, the calculations are performed at zero temperature (energy minimization), and the nanoparticles are deposited above the attractive basin in the center.

The most remarkable feature is that vertex orientations are now sometimes favorable compared to facet orientations. This is because the rough substrate presents surface hollows able to accommodate the vertex orientations: these configurations maximize the number of metal atoms in contact with the substrate. We have observed such favorable vertex orientations for the Ih and Dh structures. Furthermore, the non-truncated vertex-oriented configurations are now more favorable than the truncated Ih or Dh observed for the same potential on the flat substrate. The corresponding excess energies for the most stable orientations and truncations are shown for the three structures in Fig. 11. The results show a depression of the excess energy for the smallest Ih and Dh nanoparticles, due to the strongly favorable interaction between 
vertex orientation and surface cavities. This effect is not observed for the TOh structures, for which the (111) facet orientation is favored. For nanoparticles with less than 1400 atoms, the vertex-oriented Dh structure is the most stable, even for the smallest clusters where Ih structure could have been expected. The reason is that the Dh structure is slightly less spherical, and fits better the shape of the surface hollows.

The effect of the surface roughness is clearly important. The most striking effect is that vertexoriented, Dh structures are favored, whereas (111)-oriented TOh structures were favored on the flat surface. Note however that the effective adhesion energy on the rough surface is slightly less intense $(-0.05 \mathrm{eV} /$ atom) as explained in section III. Even with this correction, the observed stable structures on the rough surface are completely different from those given by the stability diagram shown in Fig. 6 . The effect is particularly visible for nanoparticles with lateral dimensions comparable to or smaller than the roughness of the surface. For large nanoparticles, we expect that the smooth wall approximation will tend to become more accurate. For the smallest nanoparticles, where the interaction with the roughness of the support is more crucial, further investigation in the optimized equilibrium shape and temperature effects should be performed.

\section{CONCLUSION}

We have investigated the effect of a weakly interacting amorphous support on the structural stability of silver nanoparticles. We have shown that the icosahedron/decahedron/truncated 
octahedron sequence found for free silver nanoparticles is affected by the substrate. For a smooth surface (square-well or flat atomistic silica) the truncated octahedrons deposited on their (111) facets are favored (the Dh/TOh transition is shifted to smaller clusters). Furthermore, the removal of one or more metal layers at the interface with the substrate is shown to stabilize the system. The corresponding aspect ratio has been shown to follow the Wulff-Kaishew theorem for large clusters. The introduction of nanometric roughness induces drastic changes. Vertex orientations are possible in hollows present at the surface, and decahedron structures are favored for nanoparticles smaller than 1200 atoms. These results are expected to help the interpretation of experimental data. They have been obtained for the particular silver/silica system at zero temperature, but show that the effect of the support is most probably underestimated in other similar systems. A natural perspective for this work is to investigate the effect of the temperature which is known to play an important role on thermodynamics, kinetics and nanoparticle growth. ${ }^{58,59}$

\section{Acknowledgment}

A. C. Ngandjong acknowledges a grant from the education and research ministry for his PhD. We are grateful for fruitful discussions with C. Andreazza-Vignolle, P. Andreazza and M. Benoit. This work received computational support from the French HPC resources GENCI-IDRIS (grant 2015096829) 


\section{REFERENCES}

(1) Henry, C. R. Surface studies of supported model catalysts. Surf. Sci. Rep. 1998, 31, 231325.

(2) Henry, C. R. Morphology of supported nanoparticles. Prog. Surf. Sci. 2005, 80, 92-116.

(3) Wallace, W. T.; Min, B. K.; Goodman, D. W. The nucleation, growth, and stability of oxidesupported metal clusters. Top. Catal. 2005, 34, 17-30.

(4) Freund, H.-J. Model systems in heterogeneous catalysis: selectivity studies at the atomic level. Top. Catal. 2008, 48, 137-144.

(5) Paz-Borbón, L. O.; Barcaro, G.; Fortunelli, A.; Levchenko, S. V. Aun clusters (N=1-6) supported on $\mathrm{MgO}(100)$ surfaces: Effect of exact exchange and dispersion interactions on adhesion energies. Phys. Rev. B 2012, 85, 155409.

(6) Barcaro, G.; Sementa, L.; Negreiros, F. R.; Ferrando, R.; Fortunelli, A. Interface effects on the magnetism of CoPt-supported nanostructures. Nano Lett. 2011, 11, 5542-5547.

(7) Ferrando, R.; Barcaro, G.; Fortunelli, A. Structures of small Au clusters on $\mathrm{MgO}(001)$ studied by density-functional calculations. Phys. Rev. B 2011, 83, 045418.

(8) Renaud, G. Oxide surfaces and metal/oxide interfaces studied by grazing incidence X-ray scattering. Surf. Sci. Rep. 1998, 32, 1-90.

(9) Pauwels, B.; Van Tendeloo, G.; Bouwen, W.; Theil Kuhn, L.; Lievens, P.; Lei, H.; Hou, M. Low-energy-deposited Au clusters investigated by high-resolution electron microscopy and molecular dynamics simulations. Phys. Rev. B 2000, 62, 10383-10393.

(10) Campbell, C. T. Ultrathin metal films and particles on oxide surfaces: structural, electronic and chemisorptive properties. Surf. Sci. Rep. 1997, 27, 1-111. 
(11) Hall, B. D.; Flüeli, M.; Monot, R.; Borel, J. P. Multiply twinned structures in unsupported ultrafine silver particles observed by electron diffraction. Phys. Rev. B 1991, 43, 3906-3917.

(12) Robach, O.; Renaud, G.; Barbier, A. Structure and morphology of the $\mathrm{Ag} / \mathrm{MgO}(001)$ interface during in situ growth at room temperature. Phys. Rev. B 1999, 60, 5858-5871.

(13) Graoui, H.; Giorgio, S.; Enry, C. R. Effect of the interface structure on the hightemperature morphology of supported metal clusters. Phil. Mag. B 2001, 81, 1649-1658.

(14) Vervisch, W.; Mottet, C.; Goniakowski, J. Theoretical study of the atomic structure of Pd nanoclusters deposited on a MgO(100) surface. Phys. Rev. B 2002, 65, 245411.

(15) Goniakowski, J.; Mottet, C. Palladium nano-clusters on the $\mathrm{MgO}\left(\begin{array}{lll}1 & 0 & 0\end{array}\right)$ surface: substrateinduced characteristics of morphology and atomic structure. J. Crystal Growth 2005, 275, 29-38.

(16) Mottet, C.; Goniakowski, J. Influence of epitaxial strain on supported metal cluster shapes via atomistic simulations. J. Comput. Theor. Nanoscience 2007, 4, 326-334.

(17) Jelea, A.; Mottet, C.; Goniakowski, J.; Rossi, G.; Ferrando, R. Canonical molecular dynamics simulations for crystallization of metallic nanodroplets on $\mathrm{MgO}(100)$. Phys. Rev. B 2009, 79, 165438.

(18) Ferrando, R.; Rossi, G.; Levi, A. C.; Kuntová, Z.; Nita, F.; Jelea, A.; Mottet, C.; Barcaro, G.; Fortunelli, A.; Goniakowski, J. Structures of metal nanoparticles adsorbed on MgO(001). I. Ag and Au. J. Chem. Phys. 2009, 130, 174702.

(19) Stankic, S.; Cortes-Huerto, R.; Crivat, N.; Demaille, D.; Goniakowski, J.; Jupille, J. Equilibrium shapes of supported silver clusters. Nanoscale 2013, 5, 2448-2453.

(20) Penuelas, J.; Andreazza, P.; Andreazza-Vignolle, C.; Tolentino, H. C. N.; De Santis, M.; Mottet, C. Controlling structure and morphology of CoPt nanoparticles through dynamical or static coalescence Effects. Phys. Rev. Lett. 2008, 100, 115502. 
(21) Penuelas, J.; Andreazza-Vignolle, C.; Andreazza, P.; Ouerghi, A.; Bouet, N. Temperature effect on the ordering and morphology of CoPt nanoparticles. Surf. Sci. 2008, 602, 545-551.

(22) Ewing, C. S.; Bhavsar, S.; Veser, G.; McCarthy, J. J.; Johnson, J. K. Accurate amourphous silica surface models from first-principles thermodynamics of surface dehydroxylation. Langmuir 2014, 30, 5133-5141.

(23) Zhuravlev, L. T. The surface chemistry of amorphous silica. Zhuravlev model. Colloids Surf. A: Physicochem. Eng. Aspects 2000, 173, 1-38.

(24) Tsuneyuki, S.; Tsukada, M.; Aoki, H.; Matsui, Y. First - principles interatomic potential of silica applied to molecular dynamics. Phys. Rev. Lett. 1988, 61, 869-872.

(25) van Beest, B. W. H.; Kramer, G. J.; van Santen, R. A. Force fields for silicas and aluminophosphates based on ab initio calculations. Phys. Rev. Lett. 1990, 64, 1955-1958.

(26) Guissani, Y.; Guillot, B. A numerical investigation of the liquid-vapor coexistence curve of silica. J. Chem. Phys. 1996, 104, 7633-7644.

(27) Cruz-Chu, E.; Aksimentiev, A.; Schulten, K. Water-silica force field for simulating nanodevices. J. Phys. Chem. B 2006, 110, 21497-21508.

(28) Allan, D. C.; Teter, M. P. Nonlocal pseudopotentials in molecular-dynamical densityfunctional theory: Application to SiO2. Phys. Rev. Lett. 1987, 59, 1136-1139.

(29) Lee, C.; Gonze, X. Lattice dynamics and dielectric properties of $\mathrm{SiO}_{2}$ stishovite. Phys. Rev. Lett. 1994, 72, 1686-1689.

(30) Liu, F.; Garofalini, S. H.; King-Smith, R. D.; Vanderbilt, D. First-principles studies on structural properties of beta-cristobalite. Phys. Rev. Lett. 1993, 70, 2750-2753.

(31) Rozanska, X.; Delbecq, F.; Sautet, P. Reconstruction and stability of beta-cristobalite 001, 101, and 111 surfaces during dehydroxylation. Phys. Chem. Chem. Phys. 2010, 12, 14930-14940. 
Benoit, M.; Ispas, S.; Jund, P.; Jullien, R. Model of silica glass from combined classical and ab initio molecular-dynamics simulations. Eur. Phys. J. B 2000, 13, 631-636.

(33) Van Ginhoven, R. M.; Jónsson, H.; Corrales, L. R. Silica glass structure generation for $a b$ initio calculations using small samples of amorphous silica. Phys. Rev. B 2005, 71, 024208.

(34) Tielens, F.; Gervais, C.; Lambert, J. F.; Mauri, F.; Costa, D. Ab initio study of the hydroxylated surface of amorphous silica: a representative model. Chem. Mater. 2008, 20, 3336-3344.

(35) Sherry, L. J.; Chang, S.-H.; Schatz, G. C.; Van Duyne, R. P.; Wiley, B. J.; Xia, Y. Localized surface plasmon resonance spectroscopy of single silver nanocubes. Nano Lett. 2005, 5, 2034-2038.

(36) Atwater, H. A.; Polman, A. Plasmonics for improved photovoltaic devices. Nat. Mater. 2010, 9, 205-213.

(37) Seney, C. S.; Gutzman, B. M.; Goddard, R. H. Correlation of size and surface-enhanced raman scattering activity of optical and spectroscopic properties for silver nanoparticles. J. Phys. Chem. C $2009,113,74-80$.

(38) Haes, A. J.; Zou, S.; Schatz, G. C.; Van Duyne, R. P. A nanoscale optical biosensor: the long range distance dependence of the localized surface plasmon resonance of noble metal nanoparticles. J. Phys. Chem. B 2004, 108, 109-116.

(39) Stillinger, F. H. Exponential multiplicity of inherent structures. Phys. Rev. E 1999, 59, 48.

(40) Metropolis, N.; Rosenbluth, A. W.; Rosenbluth, M. N.; Teller, A. N.; Teller, E. Equation of state calculations by fast computing machines. J. Chem. Phys. 1953, 21, 1087-1092.

(41) Rosato, V.; Guillope, M.; Legrand, B. Thermodynamical and structural properties of f.c.c. transition metals using a simple tight-binding model. Phil. Mag. A 1989, 59, 321-336.

(42) Mottet, C.; Goniakowski, J.; Baletto, F.; Ferrando, R.; Treglia, G. Modeling free and supported metallic nanoclusters: structure and dynamics. Phase Transitions 2004, 77, 101-113. 
(43) Goniakowski, J.; Finocchi, F.; Noguera, C. Polarity of oxide surfaces and nanostructures. Rep. Prog. Phys. 2008, 71, 016501.

(44) Eustathopoulos, N.; Drevet, B. Determination of the nature of metal-oxide interfacial interactions from sessile drop data. Mater. Sci. Eng. A 1998, 249, 176-183.

(45) Eustathopoulos, N.; Drevet, B. Interfacial bonding, wettability and reactivity in metal/oxide systems. J. Phys. III (France) 1994, 4, 1865-1881.

(46) Vakula, N. I.; Kuramshina, G. M.; Pentin, Y. A. Molecular simulation of interactions between silver clusters and an $\alpha$-quartz surface. Russ. J. Phys. Chem A 2013, 87, 296-302.

(47) Nagao, K.; Neaton, J. B.; Ashcroft, N. W. First-principles study of adhesion at $\mathrm{Cu} / \mathrm{SiO} 2$ interfaces. Phys. Rev. B 2003, 68, 125403.

(48) Kresse, G.; Hafner, J. Ab initio molecular dynamics for liquid metals. Phys. Rev. B 1993, $47,558-561$.

(49) Kresse, G.; Joubert, D. From ultrasoft pseudopotentials to the projector augmented-wave method. Phys. Rev. B 1999, 59, 1758-1775.

(50) Perdew, J. P.; Burke, K.; Ernzerhof, M. Generalized gradient approximation made simple. Phys. Rev. Lett. 1996, 77, 3865-3868.

(51) Morita, M.; Ohmi, T.; Hasegawa, E.; Kawakami, M.; Ohwada, M. Growth of native oxide on a silicon surface. J. Appl. Phys. 1990, 68, 1272-1281.

(52) Legrand, A. P. The surface properties of silicas; John Wiley \& Sons Ltd: Chichester, 1998.

(53) Mackay, A. L. A dense non-crystallographic packing of equal spheres. Acta Cryst. 1962, $15,916-918$.

(54) Marks, L. D. Surface structure and energetics of multiply twinned particles. Phil. Mag. A $1984,49,81-93$.

(55) Ino, S. Stability of multiply-twinned particles. J. Phys. Soc. Japan 1969, 27, 941-953. 
(56) Baletto, F.; Ferrando, R.; Fortunelli, A.; Montalenti, F.; Mottet, C. Crossover among structural motifs in transition and noble-metal clusters. J. Chem. Phys. 2002, 116, 3856-3863.

(57) Kaishew, R. Arbeitstagung Festkörperphysik, Dresden 1952, 81.

(58) Baletto, F.; Mottet, C.; Ferrando, R. Microscopic mechanisms of the growth of metastable silver icosahedra. Phys. Rev. B 2001, 63, 155408.

(59) Doye, J. P. K.; Calvo, F. Entropic effects on the size dependence of cluster structure. Phys. Rev. Lett. 2001, 86, 3570-3573. 
Table 1: DFT calculations of the adhesion energy found for isolated, single monolayer or 4 layers of silver deposited on the two cristobalite surfaces considered in this work (see text).

\begin{tabular}{|c|c|c|}
\hline & $\mathrm{SiO}_{2}-\mathrm{H}\left(7.2 \mathrm{OH} / \mathrm{nm}^{2}\right)$ & $\mathrm{SiO}_{2}-\mathrm{H}\left(3.6 \mathrm{OH} / \mathrm{nm}^{2}\right)$ \\
\hline Isolated atom & $-0.05 \mathrm{eV} / \mathrm{at}$ & $-0.16 \mathrm{eV} / \mathrm{at}$ \\
\hline 1 monolayer & $+0.01 \mathrm{eV} / \mathrm{at}$ & $-0.04 \mathrm{eV} / \mathrm{at}$ \\
\hline 4 layers & --- & $-0.04 \mathrm{eV} / \mathrm{at}$ \\
\hline
\end{tabular}


Figure 1

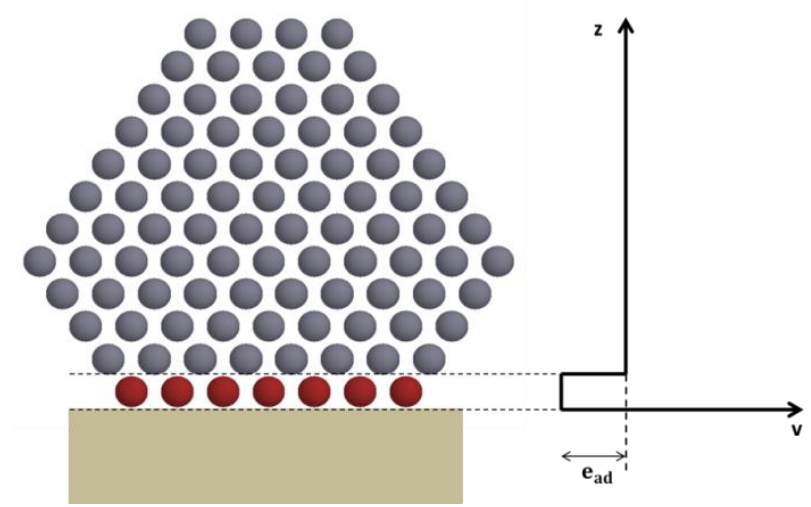

Figure 1: Schematic representation of a supported nanoparticle in the smooth-wall approximation: the substrate is modeled by a uniform square-well potential whose depth is given by the adhesion energy per surface atom, and its range corresponds to the thickness of the first atomic layer (highlighted in red). 

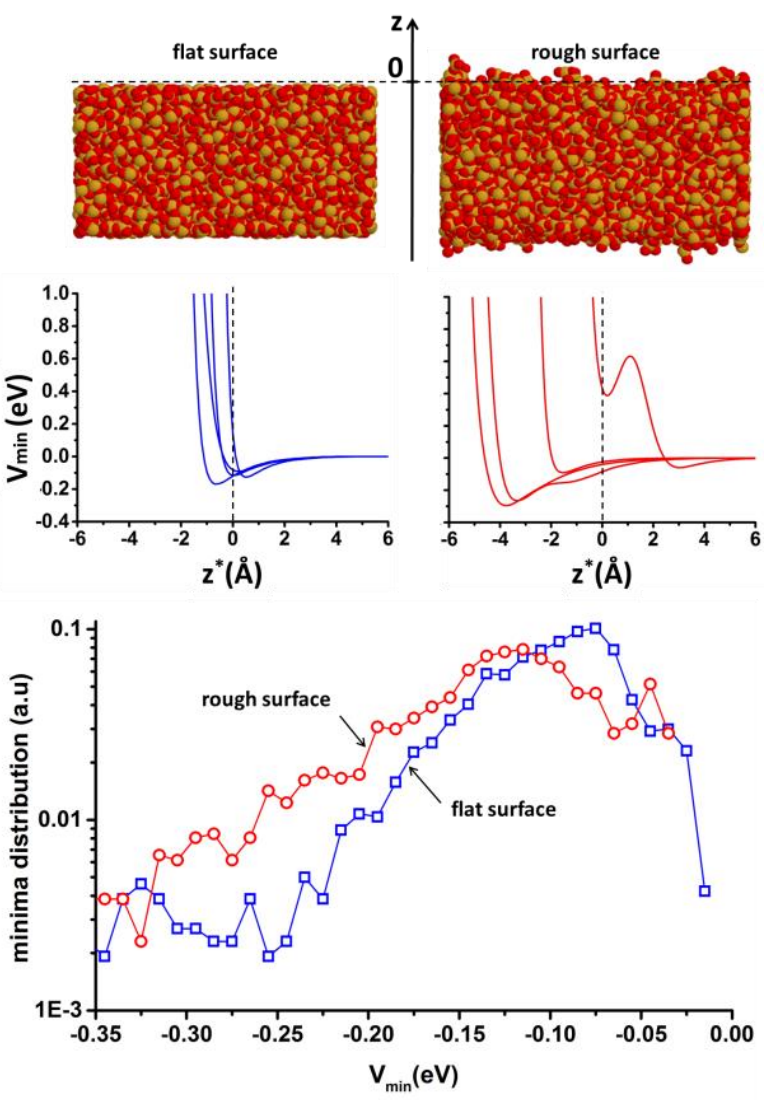

Figure 2: Upper panel: atomistic configurations of amorphous silica supports used in this work, cut from a piece of bulk amorphous silica. Left: the system is relaxed at $300 \mathrm{~K}$ (the surface remains flat). Right: the system is first melted at $2000 \mathrm{~K}$, and then cooled down to $300 \mathrm{~K}$ (the surface develops a roughness). The potential felt by a silver atom versus its height $z$ above the substrate is given below the corresponding configurations, for various positions on the surface. The distribution of the associated minima is given in the lower panel for both surfaces. 
Figure 3
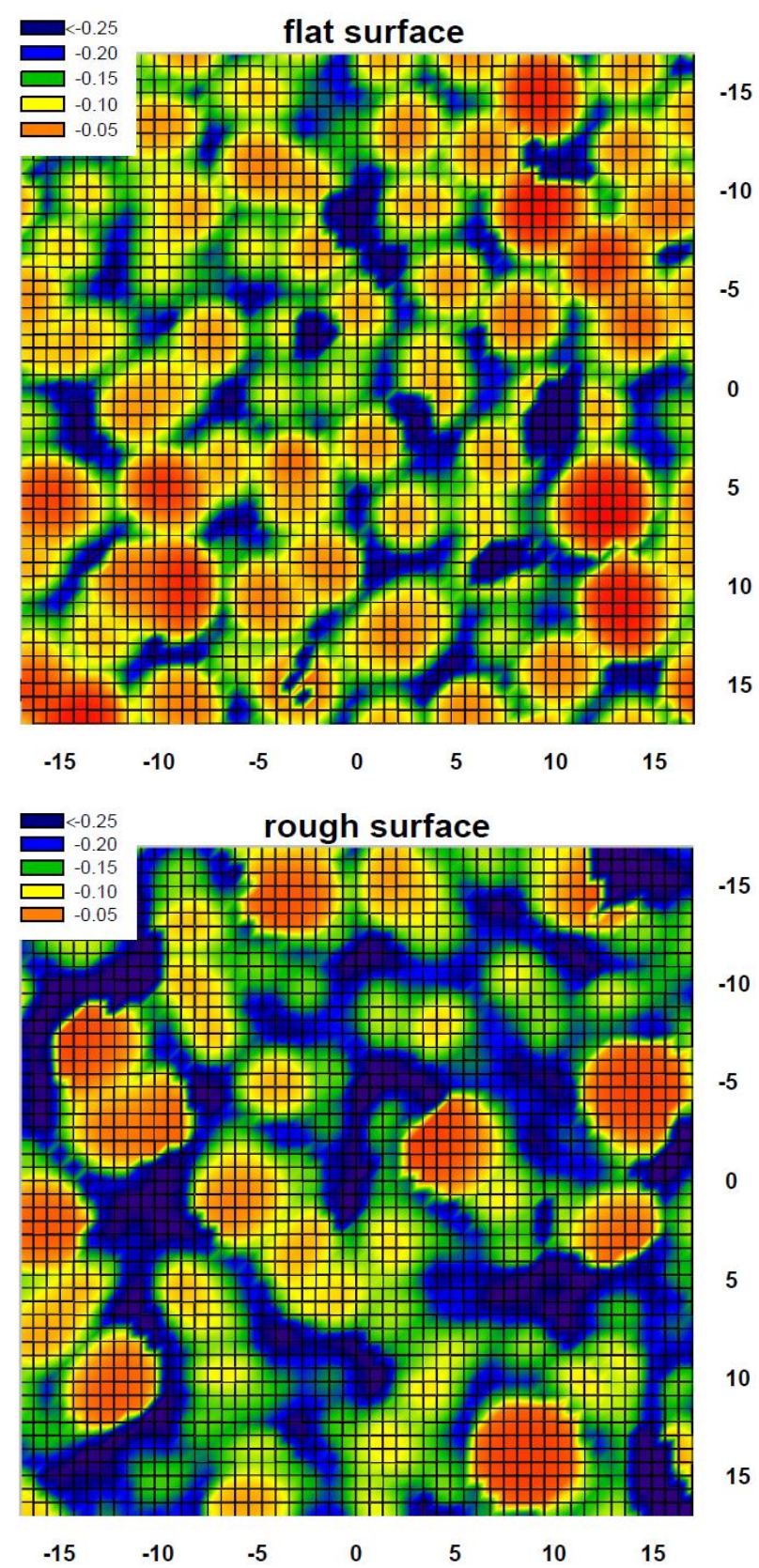

Figure 3: Two-dimensional cartography of the adsorption sites on both the flat and rough surfaces. The adsorption intensity is given in color-scale in the inset in eV/at. The $\mathrm{x}$ and $\mathrm{y}$ axes are in $\AA$. 
Figure 4

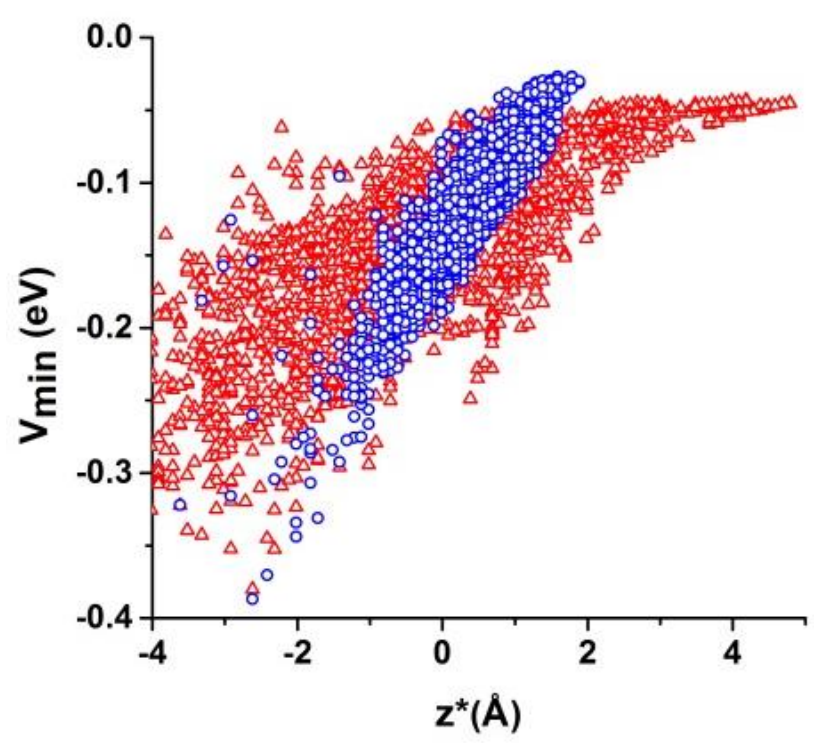

Figure 4: Correlation diagram between the depth of the silver-silica potential and the corresponding height above the surface (see text). Blue circles: flat surface. Red triangles: rough surface. 
Figure 5
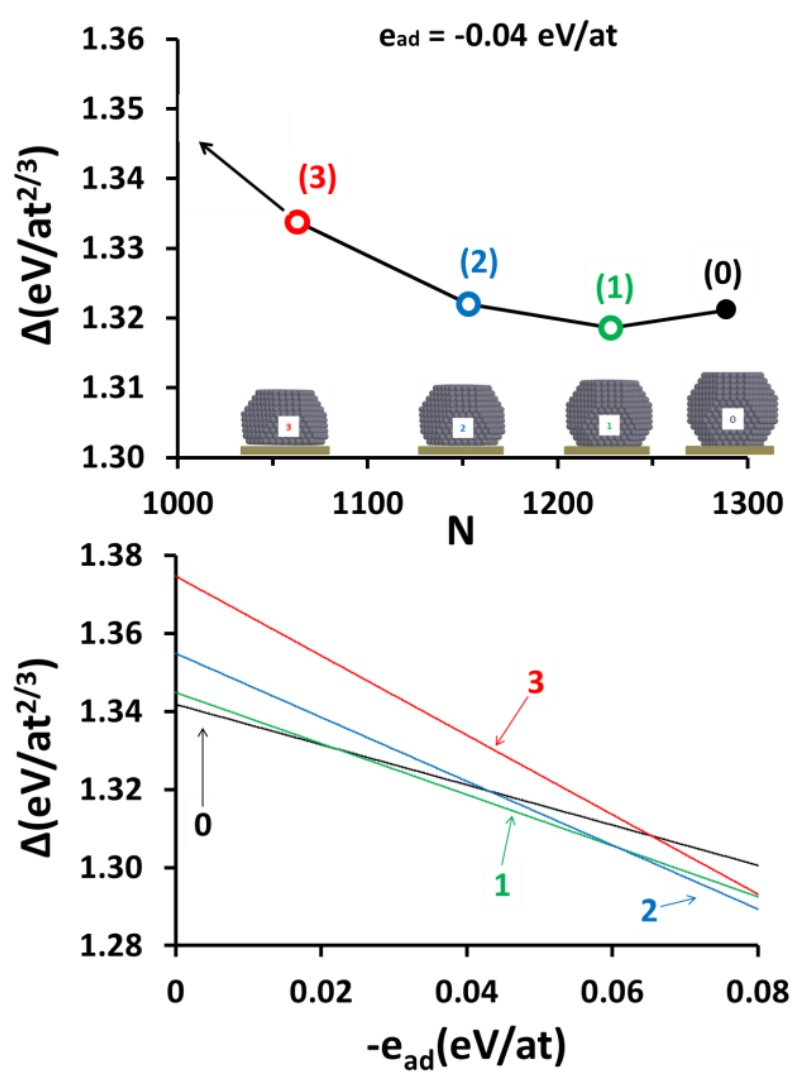

Figure 5: Upper panel: excess energy $\Delta$ for the truncated octahedron supported on the smooth surface (square well, see Fig. 1), with an adhesion energy of $-0.04 \mathrm{eV} /$ atom. Successive truncations corresponding to the removal of 0 to 3 metal layers at the interface are considered (labeled 0 to 3). The corresponding numbers of atoms are 1289, 1228, 1153 and 1063 . The arrow indicates that more truncations lead to higher energies (not shown). Lower panel: variations of the excess energy $\Delta$ for the same nanoparticles labeled 0 to 3 as a function of the square well depth (adhesion energy). 
Figure 6

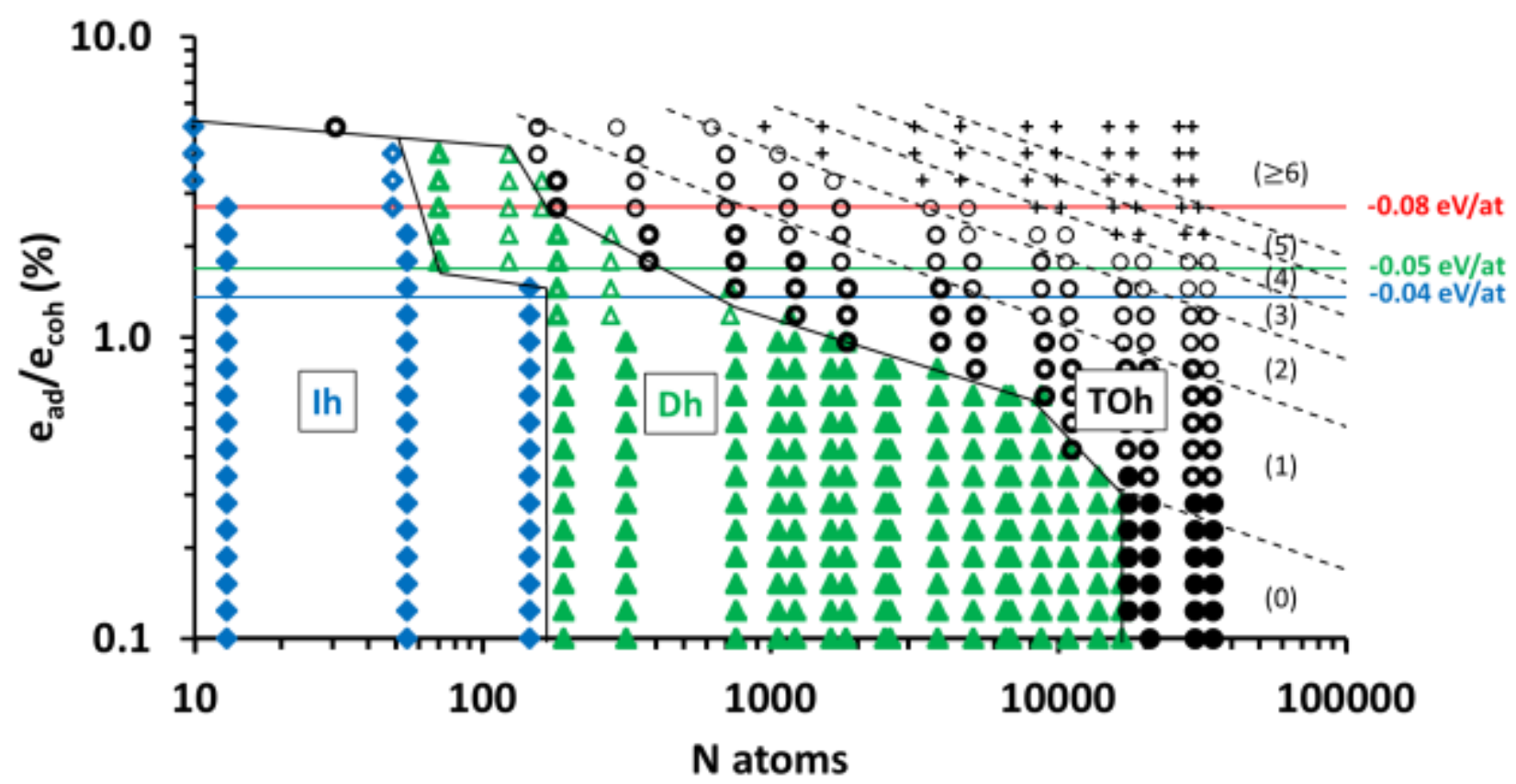

Figure 6: Diagram giving the stable configurations found by energy minimization ( $T=0 \mathrm{~K}$ ) for nanoparticles of size $\mathrm{N}$ supported on the smooth substrate (square-well potential of depth $e_{\text {ad, }}$ given in percentage of the cohesive energy of bulk silver, $-2.95 \mathrm{eV} /$ atom). The three horizontal lines correspond to the adhesion energies found in this work given in eV/atom. Blue diamonds: icosahedron (Ih); green triangles: Marks's decahedron (Dh); black circles and crosses: Wulff fcc octahedron (TOh). Filled symbols: nanoparticles non-deformed by the surface; empty symbols: truncated nanoparticles where atomic layers have been removed at the metal-substrate interface. The lighter the symbol edge, the larger the number of removed layers (1 to 3). Above three layers (for TOh only), we have used a single symbol: crosses. Dashed lines: stability regions for the truncated TOh as given by the Wulff-Kaishew theorem. The number of removed layers is given between parentheses. 
Figure 7

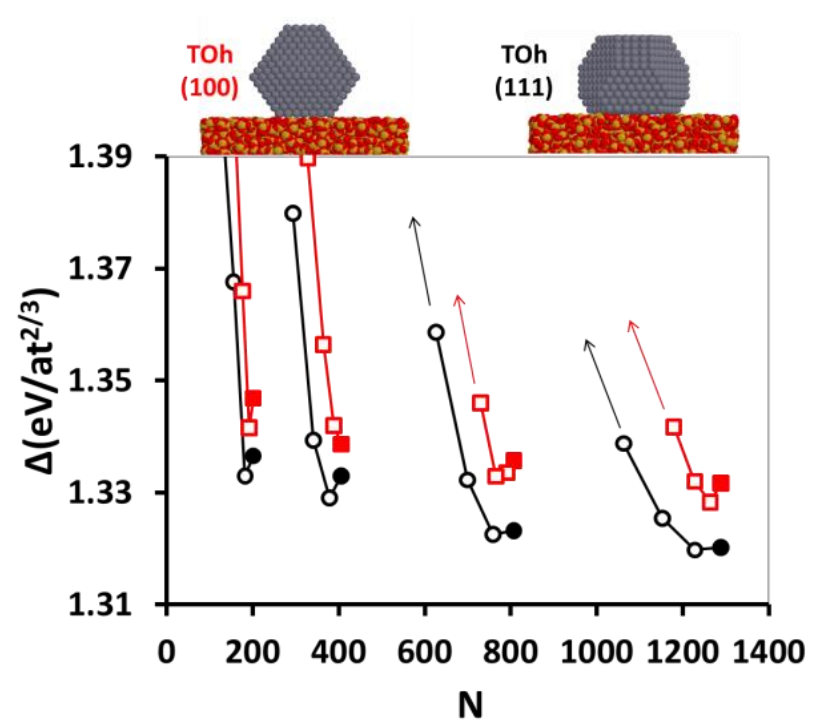

Figure 7: Excess surface energy $\Delta$ for the TOh nanoparticles of sizes $N=1289,1228,1153$ and 1063, deposited on the atomistic flat silica surface, on their (100) or (111) facet (squares and circles respectively), and their successive truncations (removal of metal layers at the interface). The $\mathrm{Ag} / \mathrm{SiO}_{2}$ potential is that fitted to the DFT calculations (giving an adhesion energy of $0.04 \mathrm{eV} /$ atom). 
Figure 8

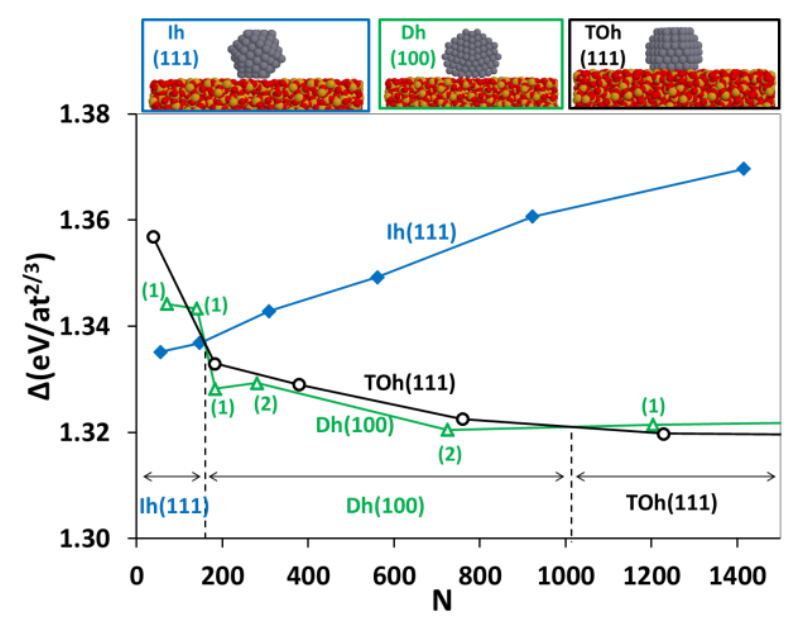

Figure 8: Excess surface energy $\Delta$ for the most stable orientation and truncation of nanoparticles supported on the atomistic flat silica surface, for a given size $\mathrm{N}$ and structure. Blue diamonds: Ih. Green triangles: truncated Dh (the number of removed layers is given between parentheses). Black circles: truncated TOh (1 layer removed). The $\mathrm{Ag} / \mathrm{SiO}_{2}$ potential is that fitted to the DFT calculations (giving an adhesion energy of $-0.04 \mathrm{eV} /$ atom). 
Figure 9

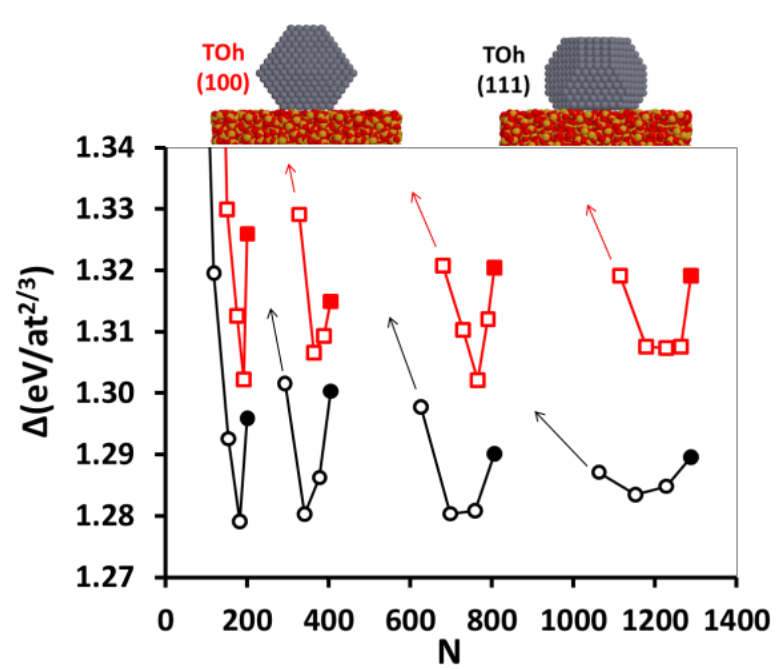

Figure 9: Excess surface energy $\Delta$ for the TOh nanoparticles of sizes $N=1289,1228,1153$ and 1063, deposited on the atomistic flat silica surface, on their (100) or (111) facet (squares and circles respectively), and their successive truncations (removal of metal layers at the interface). The $\mathrm{Ag} / \mathrm{SiO}_{2}$ potential is that fitted to the wetting angle measurements (giving an adhesion energy of $-0.08 \mathrm{eV} /$ atom). 
Figure 10

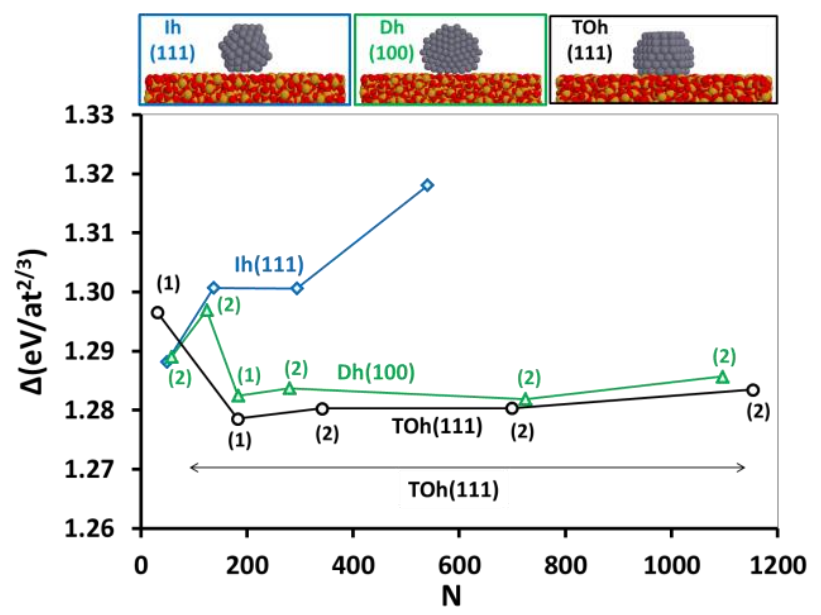

Figure 10: Excess surface energy $\Delta$ for the most stable orientation and truncation of nanoparticles supported on the atomistic flat silica surface, for a given size $\mathrm{N}$ and structure. Blue diamonds: Ih (1 layer removed). Green triangles: truncated Dh. Black circles: truncated TOh. For $\mathrm{Dh}$ and TOh the number of removed layers is given between parentheses. The $\mathrm{Ag} / \mathrm{SiO} \mathrm{S}_{2}$ potential is that fitted to the wetting angle measurements (giving an adhesion energy of $-0.08 \mathrm{eV} / \mathrm{atom}$ ). 


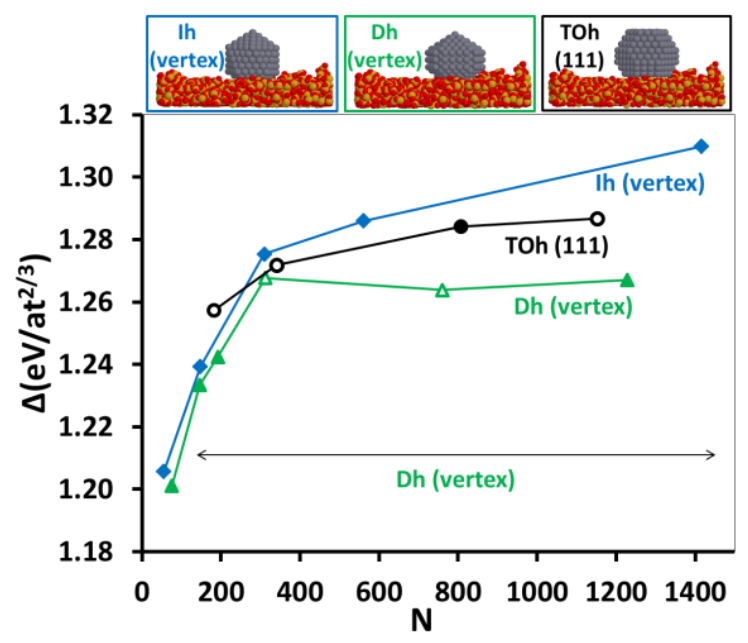

Figure 11: Excess surface energy $\Delta$ for the most stable orientation and truncation of nanoparticles supported on the atomistic rough silica surface, for a given size $\mathrm{N}$ and structure. Blue diamonds: Ih. Green triangles: Dh. Black circles: TOh. Empty symbols: truncated nanoparticles (1 layer removed). The $\mathrm{Ag} / \mathrm{SiO}_{2}$ potential is that fitted to the wetting angle measurements (giving an adhesion energy of $-0.05 \mathrm{eV} /$ atom). 


\section{TOC}

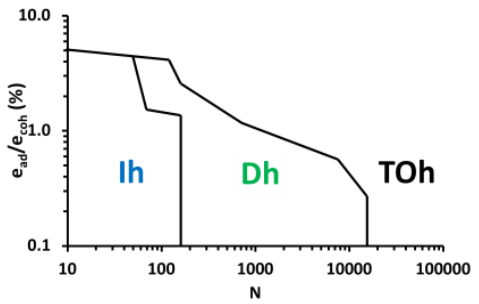

